\title{
Participação das plaquetas no processo de fibrose dos pacientes com esquistossomose mansônica
}

\author{
Participation of platelets in the process of fibrosis \\ in patients with mansonic schistosomiasis
}

\author{
Sheila Soares ${ }^{1,4,5}$, Virmondes Rodrigues Júnior², David Teixeira Nascimento Silva ${ }^{3}$ \\ e Helio Moraes-Souza ${ }^{4}$
}

\begin{abstract}
RESUMO
O objetivo deste estudo foi avaliar a ativação plaquetária através da P-selectina e o conteúdo de PDGF-AB e TGFß1, nos pacientes com esquistossomose que desenvolveram fibrose (F3), naqueles que não tiveram esta manifestação (F0) e nos controles (C). Os resultados mostraram que a percentagem de P-selectina nas plaquetas sem estímulo de agonistas foi de 10,6\% nos F3; 11,1\% nos FO, e 6,3\% nos C e após a adição de ADP/adrenalina, foi de 44\%; 25,3\% e 42\%, respectivamente. A dosagem do PDGF-AB e TGFb1 por plaquetas foi de 11,016ng/dL (F3); 3,172 $n g / d L(F 0)$ e 5,01ng/dL (C) e, (0,012ng/dL (F3); 5,27ng/dL (FO) e 4,66ng/dL (C), respectivamente. Em relação à P-selectina, concluimos que as plaquetas dos pacientes com esquistossomoses, apesar de estarem pré ativadas, mantiveram-se responsivas aos agonistas. $O$ TFG $\beta 1$ não apresentou diferença entre os três grupos, enquanto o PDGF-AB foi significantemente maior no grupo F3, sugerindo a participação deste no desenvolvimento da fibrose.
\end{abstract}

Palavras-chaves: Esquistossomose mansônica. Plaquetas. Fibrose. P-selectina. PDGF-AB. TGFß1.

\begin{abstract}
The aim of this study was to evaluate platelet activation through P-selectin, and PDGF-AB and TGF 31 content, in schistosomiasis patients who developed fibrosis (F3) and who did not present this (FO), and in a control group $(C)$. The results showed that the percentage of P-selectin in platelets without agonist stimulation was $10.6 \%$ in $\mathrm{F3}, 11.1 \%$ in $\mathrm{F0}$ and $6.3 \%$ in C. After the addition of ADP/adrenaline, the percentages were $44 \%, 25.3 \%$ and $42 \%$, respectively. The PDGF-AB and TGF 31 contents per platelet were 11,016ng/dl (F3), 3,172ng/dl (FO) and 5.01ng/dl (C) and 0,012ng/dl (F3), 5.27ng/dl (FO) and 4.66ng/dl (C), respectively. Concerning the P-selectin, we can conclude that platelets from patients with schistosomiasis continued to be responsive to agonists, despite being pre-activated. There were no differences in TGF $\beta 1$ between the groups, but the PDGF-AB content was significantly higher in F3. This suggests that PDGF-AB may have some participation in the development of fibrosis.
\end{abstract}

Key-words: Mansoni schistosomiasis. Platelet. Fibrosis. P-selectin. PDGF-AB. TGFß1.

A esquistossomose representa um dos grandes problemas de Saúde Pública acometendo, segundo a Organização Mundial de Saúde (OMS), 200 milhões de indivíduos em todo o mundo, sendo que aproximadamente 600 milhões vivem em áreas de risco, em 74 países do Globo. Dos 200 milhões de esquistossomóticos, 28,5\% dos quais infectados pelo Schistosoma mansoni, 60 milhões apresentam manifestações anatômicas e seis a 12 milhões têm manifestações clínicas da doença ${ }^{523}$. No Brasil, estima-se que cerca de seis milhões de indivíduos estejam infectados pelo Schistosoma mansoni ${ }^{15}$.
No determinismo das lesões e das formas clínicas da esquistossomose mansônica, parecem influenciar fatores ligados ao parasita e ao hospedeiro. Quanto ao primeiro, são importantes a cepa, a fase evolutiva antes e após a postura de ovos, a intensidade e o número de infestações. Do lado do hospedeiro, participam os órgãos predominantemente lesados, padrão alimentar, nutrição, alcoolismo, cor, reativação da doença, tratamento específico, constituição genética, infecções associadas (vírus da hepatite B e C e enterobactérias) e o perfil imunitário ${ }^{1821}$.

\footnotetext{
1. Curso de Pós-Graduação em Patologia, Universidade Federal do Triângulo Mineiro, Uberaba, MG. 2. Disciplina de Imunologia, Universidade Federal do Triângulo Mineiro, Uberaba, MG. 3. Disciplina de Patologia Clínica, Universidade Federal do Triângulo Mineiro, Uberaba, MG. 4. Disciplina de Hematologia e Hemoterapia, Universidade Federal do Triângulo Mineiro, Uberaba, MG. 5. Fundação Hemominas, Uberaba, MG.

Projeto financiado por auxílio da Fundação de Amparo à Pesquisa do Estado de Minas Gerais (FAPEMIG), processo n ${ }^{\circ}$ CDS 179/02.

Endereço para correspondência: Dra. Sheila Soares. Av. Dr. Getúlio Guaritá 250, 38025-240 Uberaba, MG, Brasil.

Tel: 5534 3312-5713; Fax: 5534 3312-5077

e-mail: ura.gtecnica@hemominas.mg.gov.br

Recebido para publicação em 25/5/2006

Aceito em 4/5/2007
} 
A doença apresenta-se em várias formas clínicas, com 5\% a $7 \%$ dos indivíduos desenvolvendo lesões graves, sendo que a hepatopatia representa um dos aspectos mais importantes da infecção pelo Schistossoma mansoni no homem? .

A fibrose esquistossomótica é resultado da reação granulomatosa que se desenvolve em resposta a antígenos liberados pelos ovos do Schistosoma mansoni detidos, nos pequenos ramos venosos portais ${ }^{1}$.

A participação das plaquetas no processo de fibrose hepática pode ser evidenciada inicialmente pela sua capacidade de estarem presentes no espaço de Disse. Este fato tem sido demonstrado em administração experimental de grandes doses de endotoxina, a qual promove trombocitopenia e presença de agregados plaquetários no espaço sinusoidal e conseqüentemente necrose, sendo que a remoção das plaquetas protege contra a necrose hepática focal ${ }^{11}$.

A interação das plaquetas com as células estelares em áreas necróticas tem sido evidenciada pelo achado de número aumentado de plaquetas, juntamente com leucócitos e macrófagos nestes locais ${ }^{3}$.

A participação das plaquetas na fibrose hepática possivelmente se dá através da P-selectina, Platelet-Derived Growth Factor (PDGF) AA, BB e AB e Beta Transforming Growth Factor (TGF $\beta$ ), existentes nos grânulos alfa e que são liberados quando estas são ativadas.

A P-selectina media a interação das plaquetas ativadas com neutrófilos, monócitos e alguns subtipos de células T, o que pode ser importante na inflamação e na trombose ${ }^{4}$.

O PDGF-AB pode, potencialmente, interagir com as células estelares. Pinzani e cols ${ }^{17}$, avaliando o efeito mitogênico das três formas de PDGF (AA, AB e $\mathrm{BB}$ ) nas células hepáticas de ratos, encontraram que o PDGF-BB e o PDGF-AB induzem intenso aumento na síntese de DNA e nas vias de transdução precoce de sinais relacionados ao potencial mitogênico.

Em relação ao TGF $\beta$, várias evidências sugerem que ele contribui para a doença hepática produzida pelo Schistosoma mansoni, particularmente para o acúmulo das proteínas da matriz extracelular. Kaviratne e cols ${ }^{6}$, estudando a regulação das citocinas induzidas pelo granuloma de Schistosoma mansoni e o processo de fibrose, documentaram a participação do TGF $\beta$ no desenvolvimento do granuloma e também da fibrose hepática, em resposta à infecção pelo Schistosoma mansoni. Embora as manifestações fibróticas pareçam depender dos níveis de TGF $\beta$, a fibrose se desenvolve na ausência dessa citocina.

Em decorrência das lacunas de conhecimento do processo de fibrose nos pacientes com esquistossomose e do conhecimento da participação das plaquetas na fibrose, o presente estudo foi desenvolvido com o objetivo de pesquisar eventuais diferenças quantitativas de PDGF, TFG $\beta$ e P-selectina nas plaquetas de pacientes com esquistossomose mansônica que desenvolveram fibrose, quando comparados com esquistossomóticos sem fibrose e com controles normais.

\section{MATERIAL E MÉTODOS}

O trabalho foi realizado na Disciplina de Hematologia e Hemoterapia, Hemocentro Regional de Uberaba e no Laboratório de Imunologia da Universidade Federal do Triângulo Mineiro
(UFTM). 0 presente estudo foi aprovado pelo Comitê de Ética da Fundação Hemominas, protocolado sob o número 061.

Os pacientes portadores de esquistossomose mansônica eram procedentes do Brejo do Espírito Santo, área rural do Município de Santa Maria da Vitória, sudoeste da Bahia, a 580km de Brasília.

Foram denominados de F0 aqueles pacientes que não apresentavam dados sugestivos de fibrose, tanto à avaliação clínica quanto ultra-sonográfica, realizadas entre 1976 e 2004, de acordo com padrões estabelecidos pela Organização Mundial da Saúde ${ }^{12}$. 0 grupo com fibrose intensa foi denominado de F3, sendo constituído por pacientes com clínica sugestiva de fibrose hepática, confirmada por exame ultra-sonográfico, segundo os mesmos padrões acima. Para grupo controle (indivíduos não infectados com Schistosoma mansoni), convidamos doadores de sangue, voluntários e fidelizados do Hemocentro Regional de Uberaba, zona não endêmica para esquistossomose mansônica.

Em todos os pacientes foi feita anamnese para verificação de doença hemorrágica, uso de bebida alcoólica nos últimos 10 dias, assim como uso de antiinflamatórios. Todos, após consentimento informado e assinado, foram submetidos à seguinte avaliação laboratorial:

Ativação plaquetária. Foi realizada a avaliação da ativação plaquetária através de determinação da expressão de P-selectina na superfície das plaquetas, por citrometria de fluxo, utilizando-se o anticorpo monoclonal anti-CD62-P (Immunotech ABeckman Coulter Company). Esta avaliação foi feita nas plaquetas sem ativação, após ativação com adrenalina (Aristol 1mg/mL, diluição de 1:10), ADP (Diadin - Diamed Ag, concentração 110 $\mu \mathrm{M}$ ) e adrenalina (ADR) em conjunto com ADP, nas mesmas concentrações. A leitura era feita no aparelho FACScalibur Becton Dickison, através do Software CellQuest, contando 20.000 eventos por amostra .

Liberação do conteúdo dos grânulos $\alpha$ plaquetário: PDFG-AB e TGF $\beta 1$. Para avaliar o conteúdo plaquetário do PDGF$\mathrm{AB}$ e TGF $\beta 1,400 \mu \mathrm{L}$ de plasma rico em plaquetas era estimulado, separadamente, com ADR e ADP, nas concentrações finais de $2 \mu \mathrm{M}$ e $2,5 \mu \mathrm{M}$, respectivamente. Para verificar se as plaquetas foram estimuladas, uma curva de agregação era registrada no agregômetro (Aggrometer Chrono-Log, modelo 500VS, Havertown, USA), durante 5 minutos e realizada a inspeção visual para a detecção da presença de agregado plaquetário. Posteriormente, era separado o sobrenadante e nova contagem das plaquetas era realizada neste material. A diferença entre a contagem de plaquetas do plasma rico em plaquetas(PRP) inicial e do sobrenadante correspondia ao número de plaquetas que foram ativadas e conseqüentemente liberaram o conteúdo de seus gânulos $\alpha$.

Dosagem do PDGF-AB e TGF $\beta 1$. As dosagens de PDGF-AB e TGF $\beta 1$ foram realizadas segundo as orientações do fabricante do kit ELISA Quantikine PDGF-AB Humano (R\&D Systems) e ELISA Quantikine TGF $\beta 1$ (R\&D Systems).

Análise estatística. 0 s dados foram analisados com 0 auxílio do programa Staview- version 4,57. Nas variáveis com distribuição não normal, os valores foram expressos em mediana e empregados os testes de Kruskal-Wallis, Mann-Whitney e 
Wilcoxon, considerando como estatisticamente significantes, valores menores que 0,05 .

\section{RESULTADOS}

Foram estudados 37 pacientes, 19 classificados como F0 (ausência de fibrose) e 18 como F3 (presença de fibrose). No grupo controle (C) foram estudados 12 doadores de sangue. Os grupos foram pareados por gênero e idade.

Em relação à avaliação clínica e ultra-sonográfica do fígado e baço, observamos que no grupo F0 nenhum paciente tinha esplenomegalia em três, o fígado era palpável. Porém, nenhum dos pacientes apresentou qualquer evidência de fibrose hepática à ultra-sonografia. No grupo F3, oito eram esplenectomizados, quatro tinham esplenomegalia e em seis o baço não era palpável. Quanto ao fígado, sete pacientes tinham o fígado palpável e 11 não tinham esta visceromegalia ao exame clínico. Contudo, todos tinham evidências ultra-sonográficas de fibrose grave.

0 nível de ativação plaquetária, dos três grupos estudados, determinado através da percentagem de $\mathrm{P}$-selectina liberada frente aos agonistas ADP, ADR, e ADP/ADR e representado através da mediana, pode ser observado na Tabela 1 .

Tabela 1 - Mediana da percentagem de P-selectina nas plaquetas sem adição de agonistas, ativadas com adrenalina, ADP e com o par de agonistas ADR/ADP, nos três grupos estudados.

\begin{tabular}{lcccl}
\hline Agonistas & F0 (n: 19)\% & F3 (n: 18)\% & Controle (n: 11)\% & p \\
\hline Ausente & 11,7 & 11,0 & 6,3 & 0,025 \\
ADP & 27,1 & 32,8 & 33,2 & 0,49 \\
ADR & 12,0 & 13,7 & 7,6 & 0,0064 \\
ADP/ADR & 25,3 & 44,0 & 42,0 & 0,25
\end{tabular}

FO: grupo sem fibrose; F3: grupo com fibrose; ADP: adenosina difosfato; ADR: adrenalina

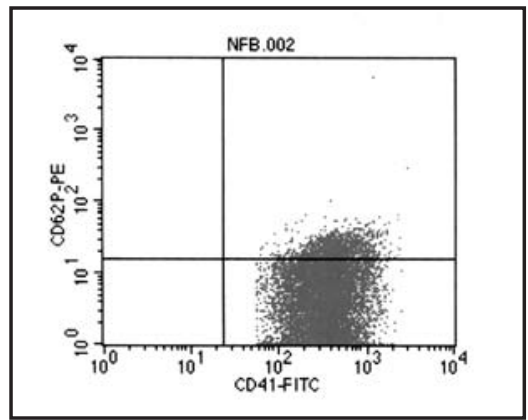

Figura 1 - Marcação de P-selectina sem adição de agonistas.

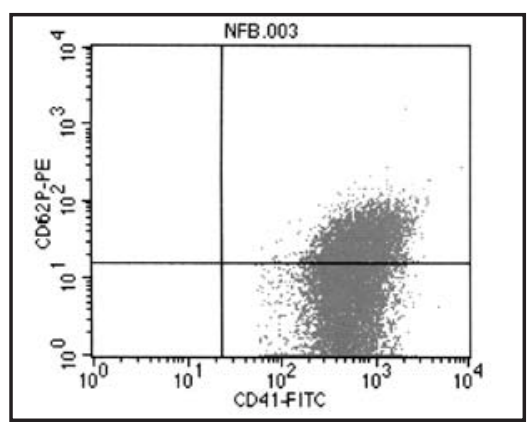

Figura 3 - Marcação de P-selectina após ativação com ADP.
As Figuras 1, 2, 3, e 4 exemplificam o comportamento da P-selectina plaquetária frente aos diferentes agonistas.

As plaquetas não ativadas por agonistas e ativadas com adrenalina mostraram percentagem de $\mathrm{P}$-selectina significativamente maior no grupo F0 e F3 em relação ao controle ( $\mathrm{p}=0,025$ e 0,0064, respectivamente). Não foi observada diferença entre os dois grupos de esquistossomóticos. Já as plaquetas ativadas com ADP e com o par de agonistas ADR/ADP apresentaram a percentagem de P-selectina maior no grupo F3, em relação ao F0. Entretanto, a diferença não foi estatisticamente significante $(\mathrm{p}=0,49$ e 0,25, respectivamente), conforme mostra a Tabela 1 .

Para verificar se existia interferência do baço no grau de ativação das plaquetas, separamos os grupos em pacientes esplenectomizados, com baço não palpável e com esplenomegalia, não sendo observado diferença estatisticamente significante entre os três grupos.

Quanto ao PDGF-AB, as medianas dos resultados das dosagens no sobrenadante do plasma (PDGF total e PDGF por plaqueta), estão representadas na Tabela 2 .

Os valores das medianas do PDGF total dos três grupos não evidenciaram diferenças estatisticamente significante. Porém, quando o cálculo foi feito por plaqueta, observamos maior liberação desse fator de crescimento no grupo F3, sendo esta diferença estatisticamente significante $(\mathrm{p}=0,0218)$.

Com relação ao TGF $\beta 1$, dosado em valores totais e por plaqueta, os valores das medianas estão apresentados na Tabela 3 .

Observamos que a mediana da dosagem do TGF $\beta 1$ no sobrenadante plaquetário, foi significativamente menor no grupo F3, (p: 0,0257). Porém, quando calculado por plaqueta, não observamos diferença significante neste grupo $(\mathrm{p}=0,1874)$.

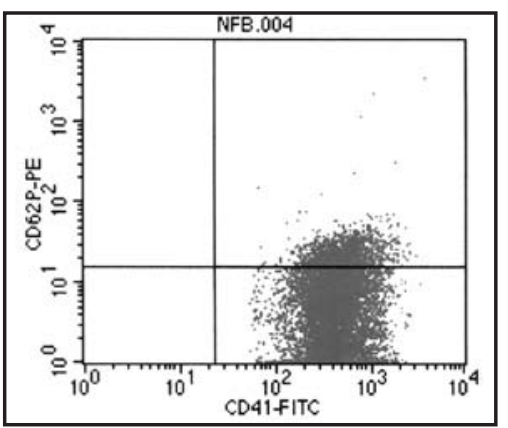

Figura 2 - Marcação de P-selectina após ativação com ADR.

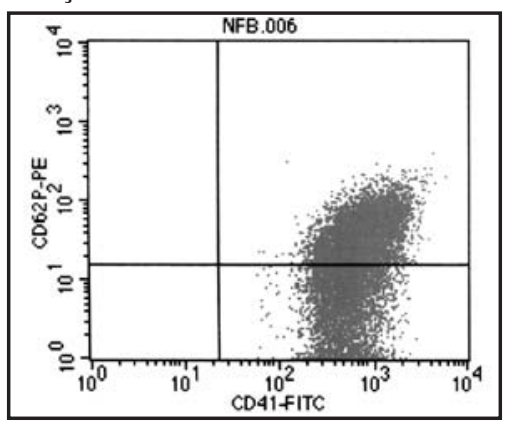

Figura 4 - Marcação de P-selectina após ativação com ADP/ADR. 
Tabela 2 - Mediana da dosagem do PDGF-AB total e por plaqueta, em ng/dL, nos três grupos estudados.

\begin{tabular}{lrrrr}
\hline & F0 (n: 19) & F3 (n: 18) & C (n: 12) & p \\
\hline PDGF total (ng/dL) & 930,0 & 1592,5 & 1052,5 & 0,1611 \\
PDGF/plaqueta (ng/dL) & 3,172 & 11,016 & 3,959 & 0,0218 \\
\hline
\end{tabular}

PDGF: Platelet-Derived Growth Factor

Tabela 3 - Mediana da dosagem de TGF $\beta 1$ total e por plaqueta, em ng/dL nos três grupos estudados.

\begin{tabular}{lrrrr}
\hline & F0 (n: 19) & F3 (n: 18) & C (n: 12) & p \\
\hline TGFß1 total (ng/dL) & 1596 & 2,84 & 7035 & 0,0257 \\
TGFß1/plaqueta ( ng/dL) & 5,27 & 0,012 & 27,745 & 0,1874
\end{tabular}

FO: grupo sem fibrose; F3: grupo com fibrose; C: grupo controle; TGF $\beta 1$ : Beta Transforming Growth Factor

\section{DISCUSSÃO}

No presente estudo, observamos que nas plaquetas não estimuladas com os agonistas, a percentagem da P-selectina foi maior nos grupos F0 e F3, aumento este estatisticamente significante. Tais achados demonstraram a existência de um estado de pré-ativação plaquetária nos pacientes portadores de esquistossomose mansônica, independente do grau de lesão hepática. Quando estimuladas com adrenalina, houve também maior expressão desta selectina nos grupos com esquistossomose em relação ao controle, mostrando diferentes respostas a este agonista nos dois grupos de pacientes. Ao serem estimuladas com ADP ou com ADP/ADR não houve diferença na expressão da Pselectina, o que nos permite aferir que estes agonistas promovem resposta similar e que, apesar da pré ativação inicial, não há lesão funcional intensa que as impeçam de responder a tais estímulos. Os nossos achados estão de acordo com os de Ogasawara e cols ${ }^{13}$ e Panasiuk e cols ${ }^{14}$.

Adicionalmente, o aumento da percentagem de P-selectina, independente da presença da fibrose (F0 ou F3), dificulta 0 entendimento da fisiopatogenia da ativação plaquetária observada nos dois grupos. Analisando o incremento de ativação plaquetária entre os três grupos, observamos que, com adrenalina, houve um aumento de $7,8 \% ; 29,8 \%$ e $21,3 \%$ nos grupos F0, F3 e controle, respectivamente e com o ADP de 144,7\%, 209,9\% e 407\%. Com o par de agonistas, ADP/ADR, observamos aumento de $128,7 \%$, $315,2 \%$ e 566,8\% nos grupos F0, F3 e controle, respectivamente. Como em nosso experimento a quantidade do agonista era a mesma para todos os grupos, a reatividade das plaquetas estaria relacionada a produção de agonista pelas plaquetas, aos receptores dos agonistas e aos mecanismos de transdução. Para compreendermos melhor este achado, seria necessário estudos adicionais avaliando tais eventos.

Em relação à função biológica da P-selectina, é evidente a sua ação reguladora na fase inicial do processo inflamatório 9 . No desenvolvimento da fibrose na esquistossomose em modelo animal, foi sugerida a necessidade de interação entre a P-selectina e o Ligante 1 da glicoproteína P-selectina (PSGL1), para diminuir a produção de citocinas inflamatórias TH2 e, conseqüientemente, diminuir o processo de fibrose, tendo sido demonstrado aumento do tamanho do granuloma e da fibrose hepática na ausência desta selectina ${ }^{22}$. Estes dados contrapõem os nossos achados, que sugerem exatamente o contrário, isto é, o grupo que não desenvolveu fibrose (F0) apresentou menor expressão de Pselectina após estímulo com os agonistas ADP/ADR.

Diante dos estudos que demonstram função profibrótica das plaquetas mediadas pela PDGF-AB e TGF $\beta 1$, na reparação tecidual de feridas e doenças vasculares oclusivas crônicas e agudas, mielofibrose e glomerulonefrite 2810161920 e da inexistência de referências desta atuação plaquetária na fibrose, procedemos a avaliação do conteúdo plaquetário destes fatores de crescimento, na tentativa de identificar aqueles que pudessem estar contribuindo com o desenvolvimento da fibrose esquistossomótica. 0 achado de valores significativamente aumentados de PDGF-AB por plaquetas, nos pacientes com fibrose, sugere que estas por terem uma maior quantidade daquele fator de crescimento nos pacientes que desenvolveram fibrose, poderiam estar estimulando, de forma mais intensa, a proliferação das células hepáticas responsáveis pela fibrogênese.

Os nossos resultados apontam para a existência de uma maior quantidade intra-plaquetária de PDGF-AB, o que poderia estar contribuindo para o processo de fibrose em pacientes com esquistossomose. Porém, estudos adicionais relacionando o grau de fibrose, a dosagem dos outros tipos de PDGF existentes nas plaquetas e a atuação desses fatores na proliferação das células de Ito, seriam necessários para a confirmação definitiva da importância do PDGF-AB na fibrogênese hepática, bem como das plaquetas como um todo, em doentes com esquistossomose mansônica.

\section{REFERÊNCIAS}

1. Boros DL, Warren KS. Delayed hypersensitivity-type granuloma formation and dermal reaction induced and elicited by a soluble factor isolated from Schistosoma mansoni eggs. Journal of Experimental Medicine 132: 488-507, 1970 .

2. Branton MH, Kopp FB. TGF-beta and fibrosis. Microbes and Infection 1:13491365, 1999.

3. Gressner AM, Bachem MG. Cellular communications and cell-matrix interactions in the pathogenesis of fibroproliferative diseases: liver fibrosis as a paradigm. Annales de Biologie Clinique 52: 2005-226, 1994.

4. Homburg SA, Mcever RP. GPM-140 mediates adhesion of stimulated platelets to neutrophilis. Blood, p.550-554, 1990

5. Jeffrey MC, Leach RM. Atlas of medical Helminthology and Protozoology, $3^{\text {a }}$ edition Endinburgh, London Melbourne and New York: Churchill Livingstone, 1991.

6. Kaviratne M, Hesse M, Leusink M, Cheever AW, Davies SJ, Mckerrow JH, Wakefield LM, Letterio JJ, Wynn TA. IL-13 Activates a mechanism of tissue fibrosis that is completely TGF- $\beta$ independent. The Journal of Immunology 173: 4020-4029, 2004 .

7. King CL. Initiation and regulation of disease in schistosomiasis. In: Mahmoud AAF (ed) Schistosomiasis. Imperial College Press, London, p. 213-64, 2001.

8. Lev PR, Marta RF, Vassallu P, Molinas FC. Variations of PDGF, TGF $\beta$, and bFGF levels in essential thrombocythemia patients treated with anagrelide. American Journal of Hematology 70: 85-91, 2002. 
9. Mannaioni PF, Di Bello MG, Manini E. Platelets and inflammation: role of plateletderived growth factor, adhesion molecules and histamine. Inflammation Research 46:4-18, 1997.

10. Martyré MC, Magdelenat H, Bryckaert MC, Laine-Bidron C, Calvo F. Increased intraplatelet levels of platelet-derived growth factor and transforming growth factor- $\beta$ in patients with myelofibrosis with myeloid metaplasia. British Journal of Haematology 77: 80-86, 1991.

11. Nakamura M, Shibazaki M, Nitta Y, Endo Y. Translocation of platelets into Disse spaces and their entry into hepatocytes in response to lipopolysaccharides interleukin-1 and tumour necrosis factor: the role of Kupffer cells. Journal of Hepatology 28: 991-999, 1998.

12. Niamey Working Group. Ultrasound in shistoso-ultrasonography for the assestement of shistosomiasis-related morbidity. World Health Organization TDR/SCH ultrason document, Geneva, Switzerland, 2000.

13. Ogasawara F, Fusegawa H, Haruki Y, Shiraishi K, Watanabe N, Matusazaki S. Platelet activation in patients with alcoholic liver disease. Tokai Journal od Experimentaland Clinical Medicine 30:41-48, 2005.

14. Panasiuk A, Prokopowiez D, Zak J, Matowicka-Karna J, Osada J, Wysocka J. Activation of blood platelets in chronic hepatitis and liver cirrhosis P-selectin Expression on Blood Platelets and Secretory Activity of $\beta$-thromboglobulin and Platelet Factor-4. Hepato-Gastroenterology 48: 818-822, 2002.

15. Passos ADC, Amaral RS. Esquistossomose mansônica aspectos epidemiológicos e de controle. Revista da Sociedade Brasileira de Medicina Tropical 31: 61-74, 1998.
16. Peters H, Eisenberg R, Daig U, Liefeldt L, Westenfeld R, Gaedeke J, Krãmer $S$, Neumayer HH. Platelet inhibition limits TGF- $\beta$ over expression and matrix expansion after induction of anti-thy 1 glomerulonephritis. Kidney International 65:2238-2248, 2004.

17. Pinzani M, Knauss TC, Pierce GF, Hsieh P, Kenney W, Dubyak GR, Abboud HE. Mitogenic signals for platelet-derived growth factor isoforms in liver fat-storing cells. American Journal of Physiology 260: 485-491, 1991.

18. Prata A. Influence of the host related factors in the development of the hepatosplenic form of schistosomiasis mansoni. Memórias do Instituto Oswaldo Cruz 87:39-44, 1992.

19. Ross R. Atherosclerosis - an inflammatory disease. The New England Journal of Medicine 340:115-126, 1999

20. Ruggeri ZM. Platelet in atherothrombosis. Nature Medicine 8:1227-1234, 2002

21. Silveira AMS, Bethony J, Gazzinelli A, Kloos H, Fraga LAO, Álvares PA, Guerra HL, Loverde PT, Oliveira RC, Gazzinelli G. High levels of IgG4 to Schistosoma mansoni egg antigens in individuals with periportal fibrosis. American Journal of Tropical Medicine and Hygiene 66: 542-549, 2002.

22. Wynn TA, Hesse M, Sandler NG, Kaviratne M, Hoffmann KF, Chiaramonte MG, Reimam R, Cheever AW, Sypek JP, Memtinkane MM. P-selectin suppresses hepatic inflammation and fibrosis in mice by regulation interferon gamma and the IL-13 decoy receptor. Hepatology 39: 676-687, 2004.

23. World Health Organization. Technical report series. The Control of Schistosomiasis 830: $1-86,1993$ 